\title{
Does high fidelity simulation training in ultrasound guided regional anaesthesia make it too difficult for novices?
}

\section{Barnes ${ }^{1}$, J. Willers ${ }^{1}$, H Rose ${ }^{2}$, B Birk ${ }^{1}$, L. Goosen ${ }^{1}$, S.Hariharan ${ }^{1}$}

${ }^{1}$ Worthing Hospital, Department of Anaesthesiology and Intensive Care; ${ }^{2}$ St. Richards Hospital, Chichester.

\section{Introduction}

What are ultrasound phantoms?

'Models' that simulate human anatomy when viewed under ultrasound

Why use phantoms?

They provide an opportunity for realistic teaching and practise of ultrasound guided regional anaesthesia (USRA) without 'practising' on patients

What are the features of a good phantom likely to include?

Realistic feel and touch on needling

Anatomical realism under ultrasound

Cost-effectiveness

- Why were we interested in evaluating phantoms now?

Recent advances in materials and methods have allowed the development of phantoms which provide more realism and complexity in terms of their feel and anatomy.

We first assessed how useful these more complex phantoms were for teaching USRA users with different levels of experience using a range of anatomical phantoms [1]

This was performed at an USRA course by assessing 'experienced' users $(n=5)$ and 'learner' users $(n=9)$. All responses from these users were positive (Likert scale responses $>80 \%$ ) [2]

In addition, the two novices that attended gave more neutral responses $(<80 \%$ but $>$ $60 \%$ ). It was unclear whether this was due to the level of difficulty, small sample number or unwillingness to give opinions due to inexperience.

We therefore decided to test the hypothesis that 'increased difficulty associated with complex high fidelity USRA phantoms could be counterproductive, and be detrimental to the learning process for novices'.

\section{Materials and method}

The phantoms evaluated:

- Interscalene and femoral (Figs. 1 and 2)

Materials of construction:

- Hetero-echoic Aqueous Dietary fibre Antifreeze Mix gel made with psyllium husk gel, water and mono ethylene glycol (ADAMgel)

- Cross-linked polyacrylamide under dynamic tension

- Anatomically suitable structures within

Evaluation technique:

- Evaluated by nine novice USRA practitioners following the same process as our previous USRA teaching course

Individual teaching stations with US images of normal anatomy and the appropriate commercial phantoms displayed as a comparison (Fig.1)

- A commercial phantom was included as a comparison. (Fig. 2, central insert)

- Feedback was gathered using a validated method with a questionnaire employing Likert scale arranged with differing numerical vales for positive responses to improve reliability [2]

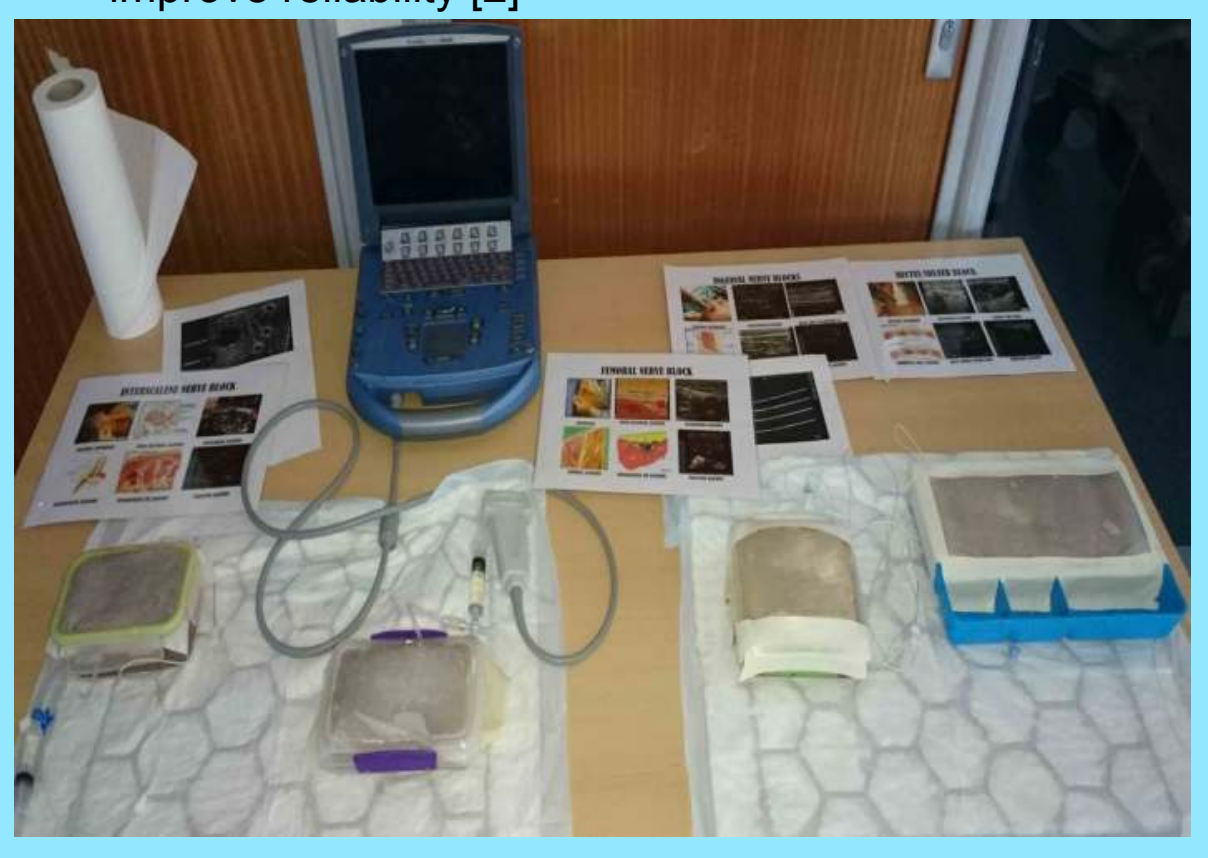

Figure 1: A teaching station with a range of custom - made ultrasound phantoms

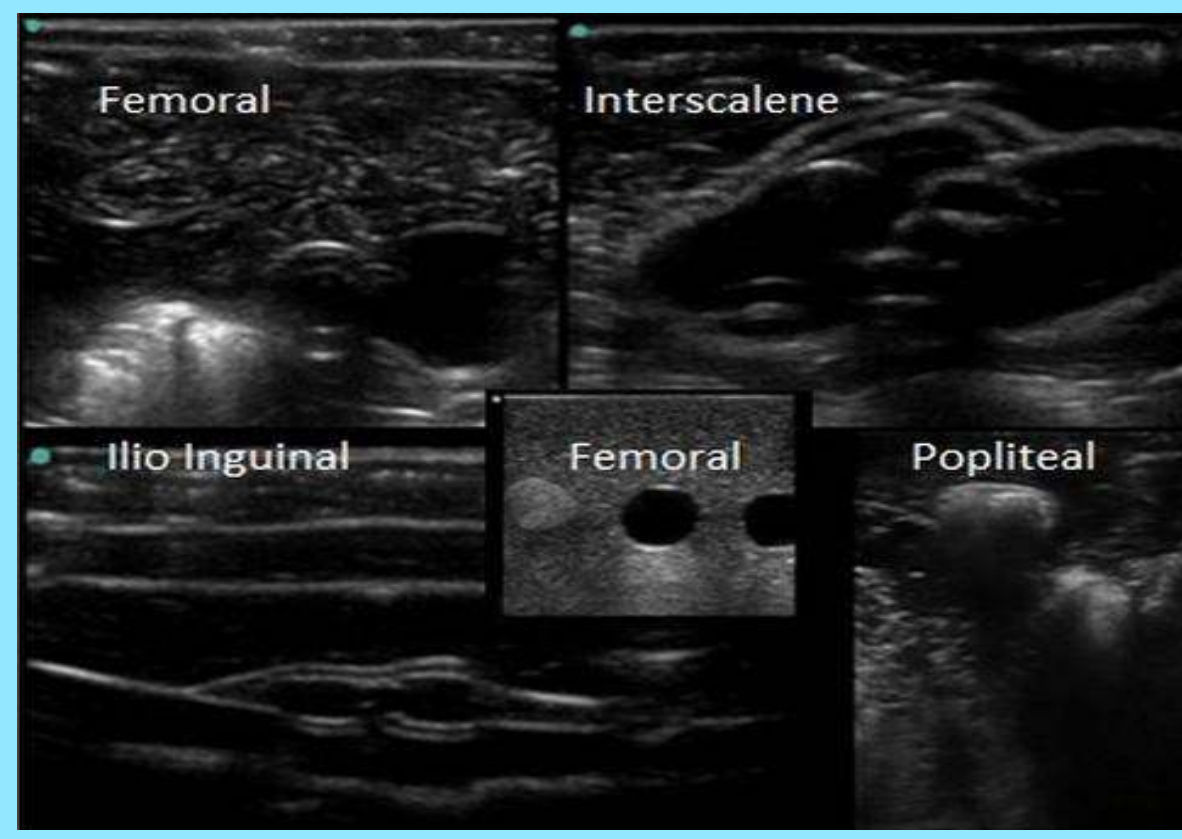

Figure 2: High fidelity USRA phantom ultrasound images

Central image is from a commercial femoral phantom (included as comparison)

\section{Results}

- The feedback from the novices group $(n=9)$ was the same in all aspects as that of the 9 (more experienced) learners (average $>80 \%$ ). [1]

Table 1: Responses from novices in USRA $(n=9)$

\begin{tabular}{ll}
\hline & $\begin{array}{c}1=\text { strongly disagree } \\
5=\text { strongly agree } \\
\text { Median score [IQR }]\end{array}$ \\
Response to question & $1[1,2]$ \\
\hline $\begin{array}{l}\text { Commercial model ultrasound image more realistic } \\
\text { than custom-made }\end{array}$ & $4[4,5]$ \\
\hline $\begin{array}{l}\text { Custom-made model ultrasound image more realistic } \\
\text { than commercial }\end{array}$ & \\
\hline Model realism aided in: & $5[5,5]$ \\
\hline Teaching technique & $4[4,5]$ \\
\hline Teaching anatomy & $5[5,5]$ \\
\hline Building confidence & $4[4,5]$ \\
\hline Increasing competence & $5[5,5]$ \\
\hline and added to the experience & $1[1,2]$ \\
\hline $\begin{array}{l}\text { Commercial model (£2500-£4000) is BETTER value } \\
\text { than the custom-made (£5-£10) }\end{array}$ & $2[2,2]$ \\
\hline Would have preferred more basic model & $2[2,2]$ \\
\hline Model realism: & $1[1,2]$ \\
\hline Distracted from teaching & $1[1,2]$ \\
\hline Makes the learning the technique too difficult & \\
\hline Did not improve the experience &
\end{tabular}

\section{Discussion}

Does high fidelity simulation training in ultrasound guided regional anaesthesia make it too difficult for novices?

Key findings:

- The custom made phantoms were widely found to be more realistic

- Overall the teaching experience was enhanced by this

In our previous study [1] the two novices gave neutral responses regarding the effect of increased realism on their teaching/learning experience

It was unclear whether this was due to:

- The realistic phantoms reproducing the higher perceived difficulty level of performing USRA for novices

Small novice sample number $(n=2)$ skewing results

- Novices feeling intimidated by the seniority of the other attendees

- Novice difficulty in giving a formed opinion due to inexperience

This separate study finds that all novices agreed or strongly agreed that the increased realism improved the learning experience.

\section{Conclusion}

There does not seem to be a negative effect caused by increased realism and difficulty associated with using complex high fidelity phantoms for USRA training for novices, thus disproving our hypothesis that the increased difficulty associated with complex high fidelity USRA phantoms could be counterproductive, and be detrimental to the learning process for novices

We believe that the realistic custom-made phantoms provide a better and costeffective alternative for safely teaching USRA.

\section{References}

[1] Varaday S, Yentis S, Clarke S. A homemade model for training in cricothyrotomy Anaesthesia 2004 ; 59: 1012-1015.

[2] Barnes L, Willers J, Rose, H, Birk, B, Goosen, L, Oosthuysen, S. Haptic realism in phantoms. A dream come true or a nightmare for learners in Ultrasound Guided Regional Anaesthesia?Anaesthesia 2015 70; 4: 29 